\title{
THE INDEX OF A NUMERICAL SEMIGROUP RING
}

\author{
OANA VELICHE
}

\begin{abstract}
Let $R=k\left[\left|t^{a}, t^{b}, t^{c}\right|\right]$ be a complete intersection numerical semigroup ring over an infinite field $k$, where $a, b, c \in \mathbb{N}$. The generalized Loewy length, which is Auslander's index in this case, is computed in terms of the minimal generators of the semigroup: $a, b$ and $c$. Examples provided show that the left hand side of Ding's inequality $\operatorname{mult}(R)-\operatorname{index}(R)-\operatorname{codim}(R)+1 \geq 0$ can be made arbitrarily large for rings $R$ with $\operatorname{edim}(R)=3$. The index of a complete intersection numerical semigroup ring with embedding dimension greater than three is also computed.
\end{abstract}

\section{INTRODUCTION}

Let $(R, \mathfrak{m}, k)$ be a local noetherian Gorenstein ring with maximal ideal $\mathfrak{m}$ and residue field $k$ and let $M$ be a finitely generated $R$-module. Auslander's delta invariant of the module $M$, denoted by $\delta(M)$, is the smallest non-negative integer $\mu$ such that there exists an exact sequence of $R$-modules, called Cohen-Macaulay approximation, $0 \rightarrow Y \rightarrow X \oplus R^{\mu} \rightarrow M \rightarrow 0$ such that $X$ is a maximal CohenMacaulay module with no free direct summands and $\operatorname{pd}_{R} Y<\infty$; see [1. It is clear from the definition that $\delta\left(R^{n}\right)=n$ for every integer $n \geq 1$. If $\operatorname{pd}_{R} M<\infty$, then $\delta(M)$ is the minimal numbers of generators of the module $M$. Moreover, a surjective homomorphism $M \rightarrow N \rightarrow 0$ induces an inequality between the delta invariants: $\delta(N) \leq \delta(M)$. In particular, $0 \leq \delta(R / \mathfrak{m}) \leq \delta\left(R / \mathfrak{m}^{2}\right) \leq \cdots \leq$ $\delta\left(R / \mathfrak{m}^{i}\right) \leq \delta\left(R / \mathfrak{m}^{i+1}\right) \leq \cdots \leq 1$, for all $i \geq 1$.

The index of the ring $R$ introduced by Auslander and studied by Ding in his thesis [5], denoted by index $(R)$, is defined as the minimum $i \geq 1$ such that $\delta\left(R / \mathfrak{m}^{i}\right)=1$; see also [6. p. 247]. The index is finite for all Gorenstein rings. Ding studies further in [5, 6] the properties of the index over Gorenstein rings with infinite residue field. He proves that a ring $R$ is regular if and only if index $(R)=1$. Moreover, he shows that the ring $R$ is a hypersurface if and only if $\operatorname{index}(R)=\operatorname{mult}(R)$, where $\operatorname{mult}(R)$ the multiplicity of the ring $R$. Furthermore, if $R$ is not regular, then

$$
\operatorname{mult}(R)-\operatorname{index}(R)-\operatorname{codim}(R)+1 \geq 0 .
$$

In particular, $\operatorname{mult}(R) \geq \operatorname{index}(R)$. Here $\operatorname{codim}(R)=\operatorname{edim}(R)-\operatorname{dim}(R)$ denotes the codimension of the ring $R$. For a proof see e.g. [6, Proposition 1.6].

Martsinkovsky [8] extends the notion of index to rings which are not necessarily noetherian, local or Gorenstein. He shows that the index is finite if $R$ is a noetherian local ring. This index satisfies all of the properties mentioned above.

Date: June 23, 2018.

2000 Mathematics Subject Classification. 16E65, 13H10, 13D05, 13P20, 13A30,18G60, 18 G99.

Key words and phrases. Cohen-Macaulay approximation, Auslander delta invariant, index, numerical semigroup ring, symmetric semigroup ring, complete intersection, Gorenstein, generalized Loewy length. 
By Ding's result [5, Theorem 2.14] when $R$ is a hypersurface the left hand side of the inequality $(*)$ is zero. Thus, one could ask the following:

Question. When $R$ is complete intersection ring is the left hand side of the inequality $(*)$ bounded above by a constant?

We answer negatively this question by providing examples which show that the left hand side can be made arbitrarily large. Example 2.5 shows that for any integer $n$ with $n \geq 2$, there exist complete intersection rings $R_{n}$ with $\operatorname{edim}\left(R_{n}\right)=3$ and

$$
\operatorname{mult}\left(R_{n}\right)-\operatorname{index}\left(R_{n}\right)-\operatorname{codim}\left(R_{n}\right)+1=2 n-3 .
$$

Proposition 3.9 shows that for any positive integer $n$ there exist complete intersection rings $R_{n}$ with $\operatorname{edim}\left(R_{n}\right)=n$ such that

$$
\operatorname{mult}\left(R_{n}\right)-\operatorname{index}\left(R_{n}\right)-\operatorname{codim}\left(R_{n}\right)+1=2^{n}-2 n .
$$

These examples were found among the numerical semigroup rings.

Working with semigroup rings requires methods different from those used in defining the index originally. In Section 1 we introduce some notions that are needed in the paper.

The main result of the paper is Theorem 2.2. It leads to an explicit formula for the index of complete intersection numerical rings semigroup rings $R=k\left[\left|t^{a}, t^{b}, t^{c}\right|\right]$, where $k$ is an infinite field and $a, b, c \in \mathbb{N}$, with $\operatorname{gcd}(a, b, c)=1$; see Remark 2.3.

Proposition 3.2 in Section 3 is a result confirmed by Bryant and Shen [3]; the proof they suggest is included here. Corollary 3.3 gives a general formula for the index of a Gorenstein numerical semigroup ring in terms of the order of certain elements of the semigroup. This corollary does not give an explicit formula for the index as given by Theorem 2.2. however its strength is illustrated in the proof of Proposition 3.9 where we compute the index for some rank 1 numerical semigroup rings with embedding dimension greater than three; see also Remarks 3.1 and 3.5 .

\section{Numerical Semigroup Rings}

In this section we introduce some terminology that will be used in this paper. Let $H$ be a semigroup minimally generated by natural numbers $a_{1}<a_{2}<\cdots<a_{e}$. The set $\mathbb{N} \backslash H$ is finite if and only if $\operatorname{gcd}\left(a_{1}, a_{2}, \ldots, a_{e}\right)=1$; in this case $H$ is called a numerical semigroup and the Frobenius number of $H$ is given by

$$
f(H)=\max \{h \notin H \mid h+i \in H, \text { for every positive integer } i\} .
$$

Note that $f(\mathbb{N})=-1$, and if $H \neq \mathbb{N}$, then $f(H)=\max \{\mathbb{N} \backslash H\}$.

1.1. The associated numerical semigroup ring of the semigroup $H$ is defined as

$$
R=k[|H|]=k\left[\left|t^{s}\right| s \in H \mid\right]
$$

and has the following properties: $R$ is a local ring with maximal ideal $\mathfrak{m}=$ $\left(t^{a_{1}}, \ldots, t^{a_{e}}\right)$, thus $\operatorname{edim}(R)=e ; R$ is a domain; $\operatorname{depth}(R)=1=\operatorname{dim}(R)$; $\operatorname{mult}(R)=a_{1}$; and $R$ is Gorenstein if and only if the semigroup $H$ is symmetric (i.e. $s \in H$ if and only if $f(H)-s \notin H$.)

A semigroup $H$ is called complete intersection or Gorenstein if the corresponding semigroup ring $R=k[|H|]$ is complete intersection or Gorenstein respectively. 
1.2. Let $R=k[|H|]$ be a Gorenstein numerical semigroup ring with $k$ infinite field. The index of $R$ is well defined and Watanabe shows in [5, Proposition 1.23] that

$$
\operatorname{index}(R)=\min \left\{i \mid \mathfrak{m}^{i} \subseteq\left(t^{s}\right) \text {, for some } s \in H\right\} .
$$

In particular, if we set $N_{s}=\min \left\{i \mid \mathfrak{m}^{i} \subseteq\left(t^{s}\right)\right\}$, for $s \in H$, then

$$
\operatorname{index}(R)=\min _{1 \leq j \leq e}\left\{N_{a_{j}}\right\} \text {. }
$$

1.3. If $(R, \mathfrak{m})$ is a noetherian local ring, the generalized Loewy length is defined by

$$
l l(R)=\min \left\{i \mid \mathfrak{m}^{i} \subseteq(\boldsymbol{x}), \text { for some system of parameters } \boldsymbol{x} \text { of } R\right\} .
$$

We recall that for any Gorenstein ring $R$ we have

$$
\operatorname{index}(R) \leq l l(R) .
$$

Indeed, if $n=l l(R)$, then $\mathfrak{m}^{n} \subseteq(\boldsymbol{x})$ for some system of parameters $\boldsymbol{x}$ of $R$. This inclusion induces a surjection $R / \mathfrak{m}^{n} \rightarrow R /(\boldsymbol{x})$ which further induces the inequality $\delta\left(R / \mathfrak{m}^{n}\right) \geq \delta(R /(\boldsymbol{x}))$. Since $\boldsymbol{x}$ is a system of parameters, we have $\operatorname{pd}_{R} R /(\boldsymbol{x})<\infty$, so $\delta(R /(\boldsymbol{x}))=1$. Thus, $\delta\left(R / \mathfrak{m}^{n}\right) \neq 0$, and by definition we have index $(R) \leq n$.

Watanabe's result from 1.2 shows that when $R$ is a Gorenstein numerical semigroup ring we have $l l(R) \leq \operatorname{index}(R)$. Therefore, the index of a Gorenstein numerical semigroup ring is given by its generalized Loewy length.

\section{The indeX of Gorenstein SEMigroup RINGS of EDIM 3}

The normal semigroup rings of the form $R=k\left[\left|t^{a}, t^{b}\right|\right]$ are hypersurfaces and $\operatorname{index}(R)=\operatorname{mult}(R)=\min \{a, b\}$. Therefore, we turn our attention to the case when the semigroup ring is of embedding dimension three. These rings were studied by Herzog in [7] and by Watanabe in [10, Proposition 3].

2.1. Let $H$ be a numerical semigroup minimally generated by three elements and set $R=k[|H|]$ for an infinite field $k$. The following are equivalent, after a possible relabeling of the generators of $H$.

(i) $R$ is complete intersection;

(ii) $R$ is Gorenstein;

(iii) There exist integers $p, x, y \geq 2$ such that $H=\langle a, b, c\rangle$ where

$a \in\langle x, y\rangle$ with $a \notin\{x, y\}$ and $b=p x, c=p y, \operatorname{gcd}(x, y)=\operatorname{gcd}(a, p)=1$.

Moreover, when one (hence all) of these cases holds, $f(H)=p x y+p a-(a+b+c)$.

Theorem 2.2. Let $R$ be a complete intersection semigroup ring of embedding dimension three. With the notation from 2.1, set $a=a^{\prime} x+a^{\prime \prime} y$ with $a^{\prime}$ and $a^{\prime \prime}$ non-negative integers. Then the following equalities hold.

(a) If $x<y$, then $N_{a}= \begin{cases}x+a^{\prime}+y \frac{a^{\prime \prime}}{x}-1, & \text { if } \frac{a^{\prime \prime}}{x} \in \mathbb{N} ; \\ y+a^{\prime}+a^{\prime \prime}+(y-x)\left\lfloor\frac{a^{\prime \prime}}{x}\right\rfloor-1, & \text { if } \frac{a^{\prime \prime}}{x} \notin \mathbb{N} .\end{cases}$ 

(b) $N_{b}= \begin{cases}p+x-1, & \text { if } a^{\prime} \neq 0 \\ & \text { or } a^{\prime}=0, \text { and } p<a^{\prime \prime} ; \\ a^{\prime \prime}+p \frac{x}{a^{\prime \prime}}-1, & \text { if } a^{\prime}=0, a^{\prime \prime}<p, \text { and } \frac{x}{a^{\prime \prime}} \in \mathbb{N} ; \\ p+x-1+\left(p-a^{\prime \prime}\right)\left\lfloor\frac{x}{a^{\prime \prime}}\right\rfloor, & \text { if } a^{\prime}=0, a^{\prime \prime}<p, \text { and } \frac{x}{a^{\prime \prime}} \notin \mathbb{N} .\end{cases}$
(c) $N_{c}= \begin{cases}p+y-1, & \text { if } a^{\prime \prime} \neq 0 \\ & \text { or } a^{\prime \prime}=0, \text { and } p<a^{\prime} ; \\ a^{\prime}+p \frac{y}{a^{\prime}}-1, & \text { if } a^{\prime \prime}=0, a^{\prime}<p, \text { and } \frac{y}{a^{\prime}} \in \mathbb{N} ; \\ p+y-1+\left(p-a^{\prime}\right)\left\lfloor\frac{y}{a^{\prime}}\right\rfloor, & \text { if } a^{\prime \prime}=0, a^{\prime}<p, \text { and } \frac{y}{a^{\prime}} \notin \mathbb{N} \text {. }\end{cases}$

Proof. (a). Set $N=N_{a}$. We may reduce to the case $a^{\prime \prime}<x$ and prove that

$$
N= \begin{cases}x+a^{\prime}-1, & \text { if } a^{\prime \prime}=0 \\ y+a^{\prime}+a^{\prime \prime}-1, & \text { if } a^{\prime \prime} \neq 0 .\end{cases}
$$

Indeed, assume that $a=a^{\prime} x+a^{\prime \prime} y$ for some non-negative integers $a^{\prime}$ and $a^{\prime \prime}$. There exist unique non-negative integers $q$ and $d^{\prime \prime}$ such that $a^{\prime \prime}=q x+d^{\prime \prime}$ with $0 \leq d^{\prime \prime}<x$. If we set $d^{\prime}=a^{\prime}+q y$, then $a=d^{\prime} x+d^{\prime \prime} y$. We have $d^{\prime \prime}=0$ if and only if $\frac{a^{\prime \prime}}{x} \in \mathbb{N}$, and then

$$
x+d^{\prime}-1=x+a^{\prime}+y \frac{a^{\prime \prime}}{x}-1 .
$$

We have $d^{\prime \prime} \neq 0$ if and only if $\frac{a^{\prime \prime}}{x} \notin \mathbb{N}$, and then

$$
y+d^{\prime}+d^{\prime \prime}-1=y+a^{\prime}+a^{\prime \prime}+(y-x)\left\lfloor\frac{a^{\prime \prime}}{x}\right\rfloor-1 .
$$

For the rest of the proof we thus assume that $0 \leq a^{\prime \prime}<x<y$. By definition, $N$ is the minimum natural number with the property that for any non-negative integers $u, v$ and $w$ such that $u+v+w=N$ there exist non-negative integers $\alpha, \beta$ and $\gamma$ with $\alpha \geq 1$ such that

$$
u a+v b+w c=\alpha a+\beta b+\gamma c .
$$

We may assume that $u=0$, thus we get

$$
v p x+w p y=\alpha a+\beta p x+\gamma p y .
$$

Since $\operatorname{gcd}(a, p)=1$, there exists an integer $\alpha^{\prime} \geq 1$ such that $\alpha=p \alpha^{\prime}$, so after dividing by $p$ the equality above becomes

$$
\begin{aligned}
v x+w y & =\alpha^{\prime}\left(a^{\prime} x+a^{\prime \prime} y\right)+\beta x+\gamma y \Longleftrightarrow \\
x\left(v-\alpha^{\prime} a^{\prime}-\beta\right) & =y\left(\gamma+\alpha^{\prime} a^{\prime \prime}-w\right) .
\end{aligned}
$$


Since $\operatorname{gcd}(x, y)=1$, there exists an integer $z$ such that

$$
\begin{aligned}
v-\alpha^{\prime} a^{\prime}-\beta & =y z \\
\gamma+\alpha^{\prime} a^{\prime \prime}-w & =x z .
\end{aligned}
$$

Setting $v=N-\delta$ and $w=\delta$ for some $\delta \in\{0,1, \ldots, N\}$, and using that $\alpha^{\prime} \geq$ $1, \beta \geq 0$ and $\gamma \geq 0$, we get that $N$ is the minimum positive integer such that for each $0 \leq \delta \leq N$, there exists $z_{\delta} \in \mathbb{Z}$ such that

$$
\frac{N-\delta-a^{\prime}}{y} \geq z_{\delta} \geq \frac{-\delta+a^{\prime \prime}}{x}
$$

In particular, for $\delta=0$ we have

$$
\frac{N-a^{\prime}}{y} \geq z_{0} \geq \frac{a^{\prime \prime}}{x}
$$

Case $a^{\prime \prime}=0$.

The inequality (2.2.1) becomes

$$
\frac{N-\delta-a^{\prime}}{y} \geq z_{\delta} \geq-\frac{\delta}{x}
$$

If we assume that $N<x$, then by choosing $\delta=N$ the inequality above implies

$$
0>-\frac{a^{\prime}}{y} \geq z_{N} \geq-\frac{N}{x}>-1,
$$

which cannot happen for any $z_{N} \in \mathbb{Z}$. Thus, $N \geq x$.

If $0 \leq \delta<x$, then $z_{\delta} \geq 0$, thus $N \geq \delta+a^{\prime}$. In particular, when $\delta=x-1$ we get

$$
N \geq x+a^{\prime}-1 \text {. }
$$

If $\delta \geq x$, write $\delta=q x+r$ with $q, r$ non-negative integers with $0 \leq r<x$. Using (2.2.4) we obtain

$$
\begin{aligned}
\frac{N-\delta-a^{\prime}}{y}+\frac{\delta}{x} & =\frac{N-a^{\prime}}{y}+\delta \frac{y-x}{x y} \\
& \geq \frac{x-1}{y}+\delta \frac{y-x}{x y} \\
& \geq \frac{x-1}{y}+\frac{y-x}{y} \\
& =1-\frac{1}{y} \\
& >1-\frac{1}{x} \\
& =\frac{x-1}{x} \\
& \geq \frac{r}{x} .
\end{aligned}
$$

So, $\frac{N-\delta-a^{\prime}}{y} \geq \frac{r-\delta}{x}=-q$. In particular, the inequality (2.2.3) holds if $z_{\delta}=-q$. Thus, we have $N=x+a^{\prime}-1$.

Case $a^{\prime \prime} \neq 0$. 
If we assume that $N<a^{\prime \prime}$, then by choosing $\delta=N$, the inequality (2.2.1) implies

$$
0 \geq-\frac{a^{\prime}}{y} \geq z_{N} \geq \frac{-N+a^{\prime \prime}}{x}>0,
$$

which is a contradiction. Thus, we must have $N \geq a^{\prime \prime}$. We apply inequality (2.2.1) to several cases of $\delta$ in order to show that we should have $N=a^{\prime}+a^{\prime \prime}+y-1$. If $\delta=a^{\prime \prime}$, then $z_{\delta} \geq 0$ and then $N \geq a^{\prime}+a^{\prime \prime}$.

If $0 \leq \delta<a^{\prime \prime}$, then $z_{\delta} \geq 1$ and then $N \geq a^{\prime}+y+\delta$. In particular, for $\delta=a^{\prime \prime}-1$,

$$
N \geq a^{\prime}+a^{\prime \prime}+y-1 \text {. }
$$

If $\delta>a^{\prime \prime}$, then write $\delta-a^{\prime \prime}=q x+r$ for non-negative integers $q, r$ and $0 \leq r<x$. Using that $N \geq a^{\prime}+a^{\prime \prime}+y-1$ and $\delta \geq a^{\prime \prime}+1$, we obtain

$$
\begin{aligned}
\frac{N-\delta-a^{\prime}}{y}+\frac{\delta}{x} & =\frac{N-a^{\prime}}{y}+\delta \frac{y-x}{x y} \\
& \geq \frac{a^{\prime \prime}+y-1}{y}+\left(a^{\prime \prime}+1\right)\left(\frac{1}{x}-\frac{1}{y}\right) \\
& =1+\frac{1}{x}-\frac{2}{y}+\frac{a^{\prime \prime}}{x} \\
& =\frac{x-1}{x}+2\left(\frac{1}{x}-\frac{1}{y}\right)+\frac{a^{\prime \prime}}{x} \\
& >\frac{r}{x}+\frac{a^{\prime \prime}}{x} .
\end{aligned}
$$

So, $\frac{N-\delta-a^{\prime}}{y} \geq \frac{r-\delta}{x}+\frac{a^{\prime \prime}}{x}=-q+\frac{a^{\prime \prime}}{x}$. Then the inequality (2.2.1) holds by taking $z_{\delta}=-q$. Therefore, $N=a^{\prime}+a^{\prime \prime}+y-1$.

(b). We consider two cases.

Case $a^{\prime} \neq 0$.

First, we show that $\mathfrak{m}^{p+x-1} \subseteq\left(t^{b}\right)$. This is equivalent with showing that for any $u, v$ and $w$ non-negative integers such that $u+v+w=p+x-1$ there exist non-negative integers $\alpha, \beta$ and $\gamma$ with $\beta \neq 0$ such that

$$
u a+v b+w c=\alpha a+\beta b+\gamma c .
$$

If $v \neq 0$, then this is clear. Assume that $v=0, u=p+x-1-\delta$ and $w=\delta$ where $\delta \in\{0, \ldots, p+x-1\}$. We consider the two cases: $\delta \leq x-1$ and $\delta>x-1$.

If $\delta \leq x-1$, we have:

$$
\begin{aligned}
u a+w c & =(p+x-1-\delta) a+\delta p y \\
& =(x-1-\delta) a+a^{\prime} p x+\left(\delta+a^{\prime \prime}\right) p y \\
& =(x-1-\delta) a+a^{\prime} b+\left(\delta+a^{\prime \prime}\right) c .
\end{aligned}
$$

Since $a^{\prime} \neq 0$, we have written $u a+w c$ in the desired format.

If $\delta>x-1$, we set $\theta=\delta-x+1>0$. Remark that $p-\theta=u \geq 0$. We have:

$$
\begin{aligned}
u a+w c & =(p+x-1-\delta) a+\delta p y \\
& =(p-\theta) a+p x y+(\theta-1) p y \\
& =(p-\theta) a+y b+(\theta-1) c .
\end{aligned}
$$


Next, we show that $\mathfrak{m}^{p+x-2} \nsubseteq\left(t^{b}\right)$. Assume that there exist non-negative integers $\alpha, \beta$ and $\gamma$ with $\beta \neq 0$ and $(p-1) a+(x-1) c=\alpha a+\beta b+\gamma c$. This is equivalent to

$$
p a+(x-1) p y=(\alpha+1) a+\beta p x+\gamma p y .
$$

Since $\operatorname{gcd}(a, p)=1$, there exists a positive integer $\alpha^{\prime}$ such that $\alpha+1=p \alpha^{\prime}$. Thus, the last equality above is equivalent to

$$
\begin{aligned}
a^{\prime} x+a^{\prime \prime} y+(x-1) y & =\alpha^{\prime}\left(a^{\prime} x+a^{\prime \prime} y\right)+\beta x+\gamma y \quad \Longleftrightarrow \\
y\left(a^{\prime \prime}-1+x-\alpha^{\prime} a^{\prime \prime}-\gamma\right) & =x\left(\alpha^{\prime} a^{\prime}+\beta-a^{\prime}\right) \Longleftrightarrow \\
y\left[x-a^{\prime \prime}\left(\alpha^{\prime}-1\right)-\gamma\right] & =x\left[a^{\prime}\left(\alpha^{\prime}-1\right)+\beta\right] .
\end{aligned}
$$

Since $\operatorname{gcd}(x, y)=1$ and $a^{\prime}\left(\alpha^{\prime}-1\right)+\beta>0$, there exists a positive integer $z$ such that

$$
x-a^{\prime \prime}\left(\alpha^{\prime}-1\right)-\gamma=x z .
$$

This together with the fact that $a^{\prime \prime}\left(\alpha^{\prime}-1\right)+\gamma \geq 0$, implies that $z=1$ thus

$$
a^{\prime \prime}\left(\alpha^{\prime}-1\right)=0 \text { and } \gamma=0 .
$$

If $a^{\prime \prime} \neq 0$, then $\alpha^{\prime}=1$ and, after dividing by $p$, the equality (2.2.5) becomes $(x-1) y=\beta x$, which is a contradiction since $\operatorname{gcd}(x, y)=1$. If $a^{\prime \prime}=0$, then $a=a^{\prime} x$ and, after dividing by $p$, the equality (2.2.5) becomes $(x-1) y=\left(\alpha^{\prime}-1\right) a^{\prime} x+\beta x$, which is again a contradiction.

Case $a^{\prime}=0$. In particular, $a^{\prime \prime} \neq 0$.

If $p<a^{\prime \prime}$, then apply part (a) with $a, b, c, p, x, y, a^{\prime}, a^{\prime \prime}$ taken to be $p x, p y, a^{\prime \prime} y, y, p, a^{\prime \prime}, x, 0$ respectively.

If $p>a^{\prime \prime}$, then apply part (a) with $a, b, c, p, x, y, a^{\prime}, a^{\prime \prime}$ taken to be $p x, a^{\prime \prime} y, p y, y, a^{\prime \prime}, p, 0, x$ respectively.

(c) follows from (b) due to the symmetry of the statement in $x$ and $y$.

Remark 2.3. Theorem 2.2 and the results from 1.2 allow us now to give a "formula" for the index of a numerical semigroup ring of embedding dimension three in terms of the generators of the semigroup. Indeed, index $(R)=\min \left\{N_{a}, N_{b}, N_{c}\right\}$, where $N_{a}, N_{b}, N_{c}$ were computed in Theorem 2.2, Below are some special cases.

Corollary 2.4. Let $R$ be a complete intersection semigroup ring of embedding dimension three. With the notation from [2.1, set $a=a^{\prime} x+a^{\prime \prime} y$ with $a^{\prime}$ and $a^{\prime \prime}$ non-negative integers and assume that $a^{\prime \prime}<x<y$.

(a) If $a^{\prime} \neq 0$ and $a^{\prime \prime} \neq 0$, then

$$
\text { index }(R)=\min \left\{y+a^{\prime}+a^{\prime \prime}-1, p+x-1\right\} .
$$

(b) If $a^{\prime}=0$ and $p<a^{\prime \prime}$, then

$$
\operatorname{index}(R)=\min \left\{y+a^{\prime \prime}-1, p+x-1\right\} .
$$

(c) If $a^{\prime \prime}=0$, then

$$
\operatorname{index}(R)=\min \left\{p+x-1, x+a^{\prime}-1\right\} .
$$

Example 2.5. Let $R=k\left[\left|t^{4 n}, t^{(4 n+1)(2 n-1)}, t^{(4 n+1)(2 n+1)}\right|\right]$ where $k$ is an infinite field and $n \geq 2$. Then $R$ is a complete intersection ring with

$$
\operatorname{mult}(R)-\operatorname{index}(R)-\operatorname{codim}(R)+1=2 n-3 .
$$


O. VELICHE

Proof. If we let $a=4 n, x=2 n-1, y=2 n+1$ and $p=4 n+1$, we obtain by Corollary 2.4 (a) that index $(R)=\min \{2 n+2,6 n-1\}=2 n+2$. So, using now 1.1 we get $\operatorname{mult}(R)-\operatorname{index}(R)-\operatorname{codim}(R)+1=4 n-(2 n+2)-2+1=2 n-3$.

\section{The INDEX OF A COMPlETE INTERSECTION SEMIGROUP RING OF EDIM $>3$}

In this section let $H=\left\langle a_{1}, a_{2}, \ldots, a_{e}\right\rangle$ be a semigroup with $\operatorname{gcd}\left(a_{1}, \ldots, a_{e}\right)=1$ and $a_{1}<a_{2}<\cdots<a_{e}$ and set $R=k[|H|]$ for an infinite field $k$. The order of an element $s$ of a semigroup $H$, see [2], is defined by

$$
\operatorname{ord}(s)=\max \left\{\sum_{i=1}^{e} \alpha_{i} \mid s=\sum_{i=1}^{e} \alpha_{i} a_{i}\right\} .
$$

Remark 3.1. If $R$ is a Gorenstein numerical semigroup ring, then 9, Lemma $2.5]$ shows that $N_{a_{1}}=\operatorname{ord}\left(f(H)+a_{1}\right)+1$. A direct computation, which we omit here, similar in difficulty and length to the proof of Theorem 2.2 , shows that when $\operatorname{edim}(R)=3$ we have $N_{a_{i}}=\operatorname{ord}\left(f(H)+a_{j}\right)+1$ for all $1 \leq j \leq 3$. Prompted by this discovery, the author consulted Bryant and Shen [3] who confirmed that a more general result holds. Although the proof they suggest uses techniques not used in this paper, we include it here as it does not appear in literature. For details on terminology and background results see [2].

Proposition 3.2. If $R=k[|H|]$ is a Gorenstein numerical semigroup ring, then

$$
N_{s}=\operatorname{ord}(f(H)+s)+1, \quad \text { for all } s \in H \backslash\{0\} .
$$

Proof. We recall, see 2, that the Apéry set of $n \in H \backslash\{0\}$ is defined by $\operatorname{Ap}(H ; n)=$ $\{w \in H \mid w-n \notin H\}$. When $s \in H$, then

$$
\operatorname{Ap}(H ; s)=\left\{w_{0}, w_{1}, \ldots, w_{s-1}\right\},
$$

where $0=w_{0}<w_{1}<\cdots<w_{s-1}=f(H)+s$.

A homogeneous element $t^{w} \in \mathfrak{m}^{i}$ belongs to $\left(t^{s}\right)$ if and only if $t^{w}=t^{s} \cdot t^{u}$ for some $u \in H$ if and only if $w-s \in H$. On the other hand, $t^{w} \in \mathfrak{m}^{i}$ if and only if $\operatorname{ord}(w) \geq i$. Therefore, we get the first equality of:

$$
\begin{aligned}
N_{s} & =\min \{i \mid \text { for all } w \in H \text { such that } \operatorname{ord}(w) \geq i, \text { we have } w \notin \operatorname{Ap}(H ; s)\} \\
& =\max \{\operatorname{ord}(w) \mid w \in \operatorname{Ap}(H ; s) \backslash\{0\}\}+1 .
\end{aligned}
$$

The second equality follows from the definition of the Apéry set. It is easy to check that the proof of [2, Proposition 3.6] holds also when $a_{1}$ is replaced by any $s \in H$. Thus, for a symmetric semigroup $H$ we obtain

$$
w_{i}+w_{j}=w_{s-1}=f(H)+s \text { for all } i+j=s-1 .
$$

In particular,

$$
\operatorname{ord}(w) \leq \operatorname{ord}(f(H)+s), \text { for all } w \in \operatorname{Ap}(H ; s) \backslash\{0\} .
$$

The desired conclusion now follows.

Corollary 3.3. If $R=k[|H|]$ is a Gorenstein numerical semigroup ring, then

$$
\operatorname{index}(R)=\min _{1 \leq j \leq e}\left\{\operatorname{ord}\left(f(H)+a_{j}\right)\right\}+1 .
$$

The next example shows that Proposition 3.2 does not hold in the case when $R$ is not a Gorenstein ring. 
Example 3.4. Let $k$ be an infinite field and $R=k\left[\left|t^{4}, t^{5}, t^{11}\right|\right]$. If $f$ is the Frobenius number of the semigroup $\langle 4,5,11\rangle$, then

$$
N_{4} \neq \operatorname{ord}(f+4)+1 \text {, }
$$

Indeed, $f=7$ and $\operatorname{ord}(f+4)+1=\operatorname{ord}(11)+1=2$ and $N_{4} \neq 2$ as $t^{10} \in \mathfrak{m}^{2} \backslash\left(t^{4}\right)$.

Remark 3.5. By contrast to Theorem 2.2, Corollary 3.3 does not give a precise formula for the index in terms of the generators of the semigroup. However, this corollary is very useful in computing the index of inductively defined Gorenstein numerical semigroup rings, at least when their Frobenius number can be explicitly computed.

Watanabe shows how one can construct complete intersection numerical semigroup rings. His result is generalized by Delorme [4, Proposition 10].

3.6. 10, Lemma 1] If $H$ is a complete intersection numerical semigroup minimally generated by natural numbers $a_{1}<a_{2}<\cdots<a_{e}$, then $H^{\prime}=\langle a, p H\rangle$ is a complete intersection for all $a$ and $p$ such that $a=\sum_{i=1}^{e} \alpha_{i} a_{i}$ with $\sum_{i=1}^{e} \alpha_{i}>1$ and $\operatorname{gcd}(a, p)=1$. Moreover, in this case we have $f\left(H^{\prime}\right)=p \cdot f(H)+(p-1) a$.

3.7. 10, Lemma 3] Set $H_{n, a}=\left\langle 2^{n}, 2^{n}+a, \ldots, 2^{n}+2^{i} a, \ldots, 2^{n}+2^{n-1} a\right\rangle$ with $n \geq 1$ and $a$ is a positive odd integer. The semigroup $H_{n, a}$ is a complete intersection as it is obtained inductively: $H_{n, a}=\left\langle 2^{n}+a, 2 H_{n-1, a}\right\rangle$, for all $n \geq 2$ and $H_{1, a}=\langle 2,2+a\rangle$.

Lemma 3.8. For $n \geq 1$, the semigroup $H_{n, a}$ defined in 3.7 has Frobenius number

$$
f\left(H_{n, a}\right)=(n-1) 2^{n}+\left(2^{n}-1\right) a .
$$

Proof. It follows by induction on $n$ using 3.6 .

Proposition 3.9. For $n \geq 1$, and $k$ an infinite field, let $R_{n, a}=k\left[\left|H_{n, a}\right|\right]$ be a semigroup ring with $H_{n, a}$ given in 3.7. Then

$$
\operatorname{index}\left(R_{n, a}\right)=n+1 \text {. }
$$

In particular,

$$
\operatorname{mult}\left(R_{n, a}\right)-\operatorname{index}\left(R_{n, a}\right)-\operatorname{codim}\left(R_{n, a}\right)+1=2^{n}-2 n .
$$

Proof. Using Corollary 3.3 it is enough to compute the minimum of ord $\left(f\left(H_{n, a}\right)+\right.$ $\left.2^{n}\right)$ and $\operatorname{ord}\left(f\left(H_{n, a}\right)+2^{n}+2^{k} a\right)$ for all $0 \leq k \leq n-1$.

We claim that $\operatorname{ord}\left(f\left(H_{n, a}\right)+2^{n}\right)=n$. Indeed, by Lemma 3.8 we have

$$
\begin{aligned}
f\left(H_{n, a}\right)+2^{n} & =n 2^{n}+\left(2^{n}-1\right) a \\
& =\left(2^{n}+a\right)+\left(2^{n}+2 a\right)+\cdots+\left(2^{n}+2^{n-1} a\right) .
\end{aligned}
$$

Thus, $\operatorname{ord}\left(f\left(H_{n, a}\right)+2^{n}\right) \geq n$. In general, we assume that

$$
f\left(H_{n, a}\right)+2^{n}=\alpha_{0} 2^{n}+\alpha_{1}\left(2^{n}+a\right)+\alpha_{2}\left(2^{n}+2 a\right)+\cdots+\alpha_{n}\left(2^{n}+2^{n-1} a\right) .
$$

This is equivalent to

$$
n 2^{n}+\left(2^{n}-1\right) a=\alpha_{0} 2^{n}+\alpha_{1}\left(2^{n}+a\right)+\alpha_{2}\left(2^{n}+2 a\right)+\cdots+\alpha_{n}\left(2^{n}+2^{n-1} a\right) .
$$

Thus, we have

$$
2^{n} \cdot\left[n-\left(\alpha_{0}+\alpha_{1}+\cdots+\alpha_{n}\right)\right]=a \cdot\left(\alpha_{1}+2 \alpha_{2}+\cdots+2^{n-1} \alpha_{n}-2^{n}+1\right),
$$


which implies, since $a$ is odd, that there exists an integer $z$ such that

$$
\begin{aligned}
n-\left(\alpha_{0}+\alpha_{1}+\cdots+\alpha_{n}\right) & =a z, \text { and } \\
\alpha_{1}+2 \alpha_{2}+\cdots+2^{n-1} \alpha_{n}-2^{n}+1 & =2^{n} z .
\end{aligned}
$$

In particular, the second equality gives that $z \geq 0$ and the first one gives that

$$
\alpha_{0}+\alpha_{1}+\cdots+\alpha_{n}=n-a z \leq n .
$$

Since the order is given by the maximum of such sums, the claim holds.

Next, we show that $\operatorname{ord}\left(f\left(H_{n, a}\right)+2^{n}+2^{k} a\right) \geq n$ for all $0 \leq k \leq n-1$, thus concluding our proof.

If $k=0$, then $f\left(H_{n, a}\right)+2^{n}+a=(n+a) 2^{n}$. Thus, ord $\left(f\left(H_{n, a}\right)+2^{n}+a\right) \geq n+a$. If $1 \leq k \leq n-2$, then

$$
\begin{aligned}
f\left(H_{n, a}\right)+2^{n}+2^{k} a & =n 2^{n}+\left(2^{n}-1\right) a+2^{k} a \\
& =\left(2^{n}+a\right)+\cdots+\left(2^{n}+2^{k-1} a\right) \\
& +2\left(2^{n}+2^{k+1} a\right)+\cdots+\left(2^{n}+2^{n-1} a\right) .
\end{aligned}
$$

Thus, $\operatorname{ord}\left(f\left(H_{n, a}\right)+2^{n}+2^{k} a\right) \geq n$.

If $k=n-1$, then

$$
\begin{aligned}
f\left(H_{n, a}\right)+2^{n}+2^{n-1} a & =n 2^{n}+\left(2^{n}-1\right) a+2^{n-1} a \\
& =\left(2^{n}+a\right)+\cdots+\left(2^{n}+2^{n-2} a\right)+(1+a) 2^{n} .
\end{aligned}
$$

Thus, $\operatorname{ord}\left(f\left(H_{n, a}\right)+2^{n}+2^{n-1} a\right) \geq n+a$. Therefore, the minimum order is $n$, so $\operatorname{index}\left(R_{n, a}\right)=n+1$. The last equality in the statement follows from 1.1

\section{ACKNOWLEDGMENTS}

I would like to thank Alex Martsinkovsky for several discussions on this subject, Lance Bryant and Yi-Huang Shen for providing the proof of Proposition 3.2 and the referee for valuable comments that improved the presentation.

\section{REFERENCES}

[1] M. Auslander, R.-O. Buchweitz, The homological theory of maximal Cohen-Macaulay approximations, Colloque en l'honneur de Pierre Samuel (Orsay, 1987). Mém. Soc. Math. France (N.S.) No. 38 (1989), 5-37.

[2] L. Bryant, Goto numbers of a numerical semigroup ring and the Gorensteiness of associated graded ring, Comm. Alg., 38 (2010), no. 6, 2092-2128.

[3] L. Bryant, Y.-H. Shen, Personal communication, (2011).

[4] C. Delorme, Sous-monoïdes d'intersection complète de N, Ann. Sci. École Norm. Sup. (4) 9 (1976), no. 1, 145-154.

[5] S. Ding, Cohen-Macaulay approximations over a Gorenstein local ring, $\mathrm{Ph}$. D. Thesis, Brandeis University (1990), 1-74.

[6] S. Ding, Cohen-Macaulay approximations and multiplicity, J. Algebra, 153 (1992), 271-288.

[7] J. Herzog, Generators and relations of abelian semigroup and semigroup rings, Manuscripta Math., 3 (1970), 175-193.

[8] A. Martsinkovsky, New homological invariants for modules over local rings, I, J. Pure Appl. Alg., 110 (1996), 1-8.

[9] Y.-H. Shen, Tangent cone of numerical semigroup rings of embedding dimension three, Comm. Alg., 39 (2011), no. 5, 1922-1940.

[10] K. Watanabe, Some examples of one dimensional Gorenstein domains, Nagoya Math. J. vol. 49 (1973), 101-109. 
Department of Mathematics, Northeastern University, 360 Huntington Avenue, Boston, MA 02115

E-mail address: o.veliche@neu.edu

$U R L:$ http://www.math.northeastern.edu/ veliche

Saint Herman Christian School, 62 Harvard Avenue, Allston, Ma 02134

E-mail address: oana@sainthermanschool.org 